\title{
Perfil epidemiológico da sífilis em gestantes no Brasil
}

\author{
Epidemiological profile of syphilis in pregnants women in Brazil
}

Perfil epidemiológico de sífilis en mujeres embarazadas en Brasil

Amanda Maués Ramos ${ }^{1 *}$, Thiago José Maués Ramos ${ }^{1}$, llka Lorena de Oliveira Farias Costa ${ }^{2}$, Ana Paula Oliva Reis ${ }^{3}$, Sérgio Beltrão de Andrade Lima ${ }^{4}$, Daniele Socorro de Brito Souza Paiva ${ }^{2,4}$.

\section{RESUMO}

Objetivo: Conhecer o número de casos e taxa de detecção de sífilis em gestantes e de sífilis congênita no Brasil, bem como o perfil dessas gestantes e o momento do diagnóstico. Métodos: Estudo ecológico, com dados referentes à sífilis em gestantes e sífilis congênita no Brasil no período de 2011 a 2020, obtidos na plataforma online de indicadores de Sífilis do Ministério da Saúde. Resultados: Foi observado um aumento gradativo da incidência de sífilis materna e consequentemente de sífilis congênita. Foram registrados 385.412 casos de sífilis em gestantes e 190.034 casos de sífilis congênita no período em estudo. A taxa de detecção de sífilis para cada 1.000 nascidos vivos foi de 4,7 em 2011 e 20,6 em 2020 em gestantes e de 3,3 em 2011 e 7,7 em 2020 em menores de um ano. A sífilis em gestantes foi mais prevalente em mulheres jovens, pardas e de baixa escolaridade. Houve um aumento gradativo no decorrer dos anos do estudo do diagnóstico materno de sífilis no pré-natal nas fases latentes e no $1^{\circ}$ trimestre. Conclusão: O Brasil ainda apresenta índices preocupantes em relação à sífilis na gestação e à sífilis congênita, podendo ser consideradas como doenças reemergentes.

Palavras-chave: Sífilis, Gestantes, Epidemiologia, Sífilis congênita.

\begin{abstract}
Objective: To know the number of cases and detection rate of syphilis in pregnant women and congenital syphilis in Brazil, as well as the profile of these pregnant women and the time of diagnosis. Methods: Ecological study, with data on syphilis in pregnant women and congenital syphilis in Brazil from 2011 to 2020, obtained from the Ministry of Health's online platform of syphilis indicators. Results: A gradual increase in the incidence of maternal syphilis and consequently of congenital syphilis was observed. There were 385,412 cases of syphilis in pregnant women and 190,034 cases of congenital syphilis in the study period. The syphilis detection rate for every 1,000 live births was 4.7 in 2011 and 20.6 in 2020 in pregnant women and 3.3 in 2011 and 7.7 in 2020 in children under one year of age. Syphilis in pregnant women was more prevalent in young, brown and low-educated women. There was a gradual increase over the years of study of maternal diagnosis of syphilis in prenatal care in latent phases and in the 1st trimester. Conclusion: Brazil still has worrying rates in relation to syphilis during pregnancy and congenital syphilis, which can be considered as reemerging diseases.
\end{abstract}

Key words: Syphilis, Pregnant women, Epidemiology, Syphilis congenital.

\footnotetext{
1 Universidade Federal do Pará (UFPA), Belém - PA. *E-mail: amandamauesr@gmail.com

2 Fundação Santa Casa de Misericórdia do Pará (FSCMP), Belém - PA.

${ }^{3}$ Secretaria de Saúde do Estado do Pará (SESPA), Belém - PA.

${ }^{4}$ Universidade do Estado do Pará (UEPA), Belém - PA.
}

SUBMETIDO EM: 12/2021

PUBLICADO EM: 1/2022 


\section{RESUMEN}

Objetivo: Conocer el número de casos y la tasa de detección de sífilis en gestantes y sífilis congénita en Brasil, así como el perfil de estas gestantes y el momento del diagnóstico. Métodos: Estudio ecológico,con datos sobre sífilis en gestantes y sífilis congénita en Brasil de 2011 a 2020, obtenido de la plataforma en línea de indicadores de sífilis del Ministerio de Salud. Resultados: Se observó un aumento paulatino de la incidencia de sífilis materna y consecuentemente de sífilis congénita. Hubo 385,412 casos de sífilis en mujeres embarazadas y 190,034 casos de sífilis congénita en el período de estudio. La tasa de detección de sífilis por cada 1.000 nacidos vivos fue de 4,7 en 2011 y de 20,6 en 2020 en mujeres embarazadas y de 3,3 en 2011 y de 7,7 en 2020 en menores de un año. La sífilis en mujeres embarazadas fue más prevalente en mujeres jóvenes, morenas y con bajo nivel educativo. Hubo un incremento paulatino a lo largo de los años de estudio del diagnóstico materno de sífilis en la atención prenatal en fases latentes y en el 1er trimestre. Conclusión: Brasil aún tiene tasas preocupantes en relación con la sífilis durante el embarazo y la sífilis congénita, que pueden ser consideradas como enfermedades reemergentes.

Palabras clave: Sífilis, Mujeres embarazadas, Epidemiología, Sífilis congénita.

\section{INTRODUÇÃO}

A sífilis na gestação ainda é observada em uma parcela significativa de mulheres, o que favorece diretamente à ocorrência de sífilis congênita (TORRES RG, et al., 2019). É uma infecção sistêmica causada pela bactéria Treponema pallidum, transmitida principalmente por via sexual e vertical, e menos frequente por contato direto no canal de parto com lesões sifilíticas e por transfusão sanguínea. Durante 0 aleitamento materno, ocorrerá transmissão apenas se houver lesão mamária por sífilis (SILVA GM, et al., 2021). A doença se divide em quatro estágios: sífilis primária, secundária, latente e tardia ou terciária, de acordo com o tempo de evolução da doença (MAGALHÃES DMS, et al., 2011).

A sífilis primária é caracterizada pela presença de cancro duro (lesão circunscrita geralmente ulcerada indolor que corresponde ao local de inoculação do treponema), dificilmente diagnosticada em mulheres devido acometer principalmente o colo uterino e raramente a vulva. A sífilis secundária reflete a disseminação hematogênica do treponema, com a presença das roséolas sifilíticas (lesões exantemáticas, generalizadas e não pruriginosas) associadas a sintomas gerais como febre baixa, astenia, mialgia, artralgia, alopecia, entre outros. A sífilis terciária é pouco frequente nos dias atuais, com a presença de lesões circunscritas não infectantes (gomas) que podem acometer principalmente o sistemas nervoso e circulatório. Entre as fases clínicas ocorre as fases latentes (precoce e tardia), somente diagnosticadas pelos testes sorológicos (MENEZES ML e PASSOS MR, 2018).

Segundo o Ministério da Saúde (MS) (2020a), a transmissão vertical ocorre quando há passagem do treponema da gestante não tratada ou tratada inadequadamente ao feto através da plac enta. Ainda que a transmissão vertical da sífilis possa ocorrer em qualquer fase gestacional ou estágio clínico, os principais fatores que determinam a probabilidade de transmissão estão relacionados à classificação clínica da sífilis na gestante e à duração da exposição do feto no útero (SARACENI V, et al., 2017).

Como a carga bacteriana circulante do T. pallidum na doença sintomática (fases primária e secundária) é maior, consequentemente o risco de transmissão também é maior (70 a 100\%). Enquanto que nas fases latentes e terciária este risco oscila entre 10 a 30\%. Ademais, quanto mais avançada for a gestação, maior é o risco de infecção fetal, visto que a permeabilidade da barreira transplacentária aumenta com a idade gestacional (MS, 2018; DANTAS LA, 2017).

A sífilis congênita constitui um dos mais graves desfechos preveníveis da gestação e responde por aproximadamente $50 \%$ de recém-nascidos com sequelas físicas, sensoriais ou de desenvolvimento (MAGALHÃES DMS, et al., 2011; CARDOSO ARP, et al., 2018). Outras consequências ao recém-nascido incluem o abortamento, natimortalidade, prematuridade, baixo peso ao nascer, morte fetal e perinatal e sequelas diversas da doença que poderão se manifestar até os 2 anos de vida (MS, 2018; CONCEIÇÃO HN, et al., 2021; LAFETÁ KRG, et al., 2016). 
A história prévia ou atual de lesão ulcerada em região genital deve ter a sífilis entre as possibilidades diagnósticas. Assim como na presença de rash cutâneo palmo-plantar ou condiloma plano a sífilis na fase secundária deve ser pensada. Entretanto a maioria das gestantes apresenta-se assintomática ao diagnóstico, sem história prévia de infecção ou tratamento, com identificação na fase latente indeterminada da doença (CARDOSO ARP, et al., 2018; LAFETÁ KRG, et al., 2016; MARQUES JVS, et al., 2018).

Dessa forma, a realização de testes laboratoriais para a identificação dessa infecção na gestação é de suma importância. Com o intuito de tratar precocemente às pacientes infectadas, o Ministério da Saúde (2018) recomenda a realização de um teste não treponêmico (VDRL ou RPR) associado a um teste treponêmico (Teste rápido, TPHA ou FTA-Abs) no início do pré-natal (preferencialmente no 1ำtrimestre) e no início do terceiro trimestre e novamente na admissão para parto ou aborto, independente dos exames anteriores.

Os testes não treponêmicos são muito sensíveis e podem ser titulados, o que auxilia na avaliação da resposta ao tratamento. Todavia, principalmente na fase latente, podem apresentar resultados falso-positivos e falso-negativos em até $2 \%$ e $25 \%$, respectivamente. Por outro lado, os testes treponêmicos são específicos para sífilis, porém persistem positivos como marca sorológica da infecção mesmo após o tratamento (TORRES RG, et al., 2019; CARDOSO AR,P et al., 2018).

Para o diagnóstico de sífilis é necessário dados clínicos, testes sorológicos, história de infecções prévias e averiguação de recente exposição. Ademais, a infecção de sífilis não confere imunidade protetora, assim como não existe vacina, desta forma, um indivíduo pode se inf ectar várias vezes (MS, 2021).

Para definição de sífilis na gravidez são aceitas três situações, durante o pré-natal, parto e/ou puerpério: Gestante assintomática com pelo menos um teste reagente (treponêmico e/ou não treponêmico com qualquer titulação) e sem registro de tratamento prévio; Gestante sintomática com pelo menos um teste reagente (treponêmico e/ou não treponêmico com qualquer titulação); Gestante com ambos os testes reagentes (treponêmico e não treponêmico com qualquer titulação), independente da história clínica de sífilis e de tratamento prévio (MENEZES ML e PASSOS MR, 2018).

As parcerias sexuais das gestantes com sífilis devem ser tratadas independente dos testes sorológicos, visto que podem estar infectadas mesmo com resultados negativos (MS, 2018). Mas não devem ser mais consideradas para o diagnóstico de sífilis congênita (MENEZES ML e PASSOS MR, 2018).

A sífilis é agravo de notificação compulsória para fins de vigilância epidemiológica, desde 2005 nas gestantes e 1986 nos casos congênitos, e apresentou um aumento considerável de casos nos últimos anos, podendo acarretar complicações ao binômio mãe-feto. A alta prevalência deste agravo está associada ao baixo nível de escolaridade materna e à falha da assistência pré-natal, o que reflete nos dados registrados pela Organização Mundial de Saúde (OMS) que apontam a maior prevalência da doença em países subdesenvolvidos (BARBOSA DRM, et al., 2018; MASCHIO-LIMA T, et al., 2019; (MENEZES ML e PASSOS MR, 2018).

Nesse sentido, considerando o impacto da sífilis em gestantes na saúde pública, este estudo visou conhecer o número de casos e taxa de detecção de sífilis em gestantes e de sífilis congênita no Brasil nos últimos 10 anos, bem como o perfil dessas gestantes e o momento do diagnóstico, a fim de identificar os pontos de vulnerabilidade da assistência à saúde da mulher e do recém-nascido.

\section{MÉTODOS}

Trata-se de um estudo ecológico, com dados referentes à sífilis em gestantes no Brasil nos últimos 10 anos, no período de 2011 a 2020, obtidos na plataforma online Indicadores e Dados Básicos da Sífilis nos Municípios Brasileiros do Departamento de Doenças de Condições Crônicas e Infecções Sexualmente Transmissíveis (DCCl) do MS, com base no Sistema de Informação de Agravos de Notificação (SINAN). As variáveis investigadas foram: número de casos notificados e taxa de detecção de sífilis em gestantes e de sífilis congênita, faixa etária, escolaridade, cor ou raça, idade gestacional, classificação clínica e tratamento das gestantes com sífilis. 
Para análise dos dados, os mesmos foram organizados em tabelas e gráficos do prog rama Microsoft Office Excel 2016 e Microsoft Word 2016. Por utilizar uma plataforma online do Ministério da Saúde, cujos dados se encontram disponíveis para livre acesso, este estudo não demandou aprovação por comitê de ética em pesquisa com seres humanos.

\section{RESULTADOS}

Nos últimos 10 anos, 385.412 casos de sífilis em gestantes foram notificados, sendo observado um crescimento de 459,9\% do número de casos entre os anos 2011 ( $n=13.752)$ e 2018 ( $n=63.250)$, com uma discreta redução em 2019 ( $n=62.086)$ e 2020 ( $n=61.441)$. Como consequência, foi observado também um aumento dos casos de sífilis congênita, que apresentou as mesmas variações que as gestantes ao longo dos anos. Foram notificados 190.034 casos de sífilis congênita em menores de um ano no período em estudo, com um aumento de 278,9\% entre os anos 2011 ( $n=9.488)$ e 2018 ( $n=26.464)$, e com diminuição em 2019 $(n=24.236)$ e $2020(n=22.065)$ (Gráfico 1).

Gráfico 1 - Número de casos de sífilis em gestantes e de sífilis congênita em menores de um ano de idade por ano. Brasil, 2011-2020.

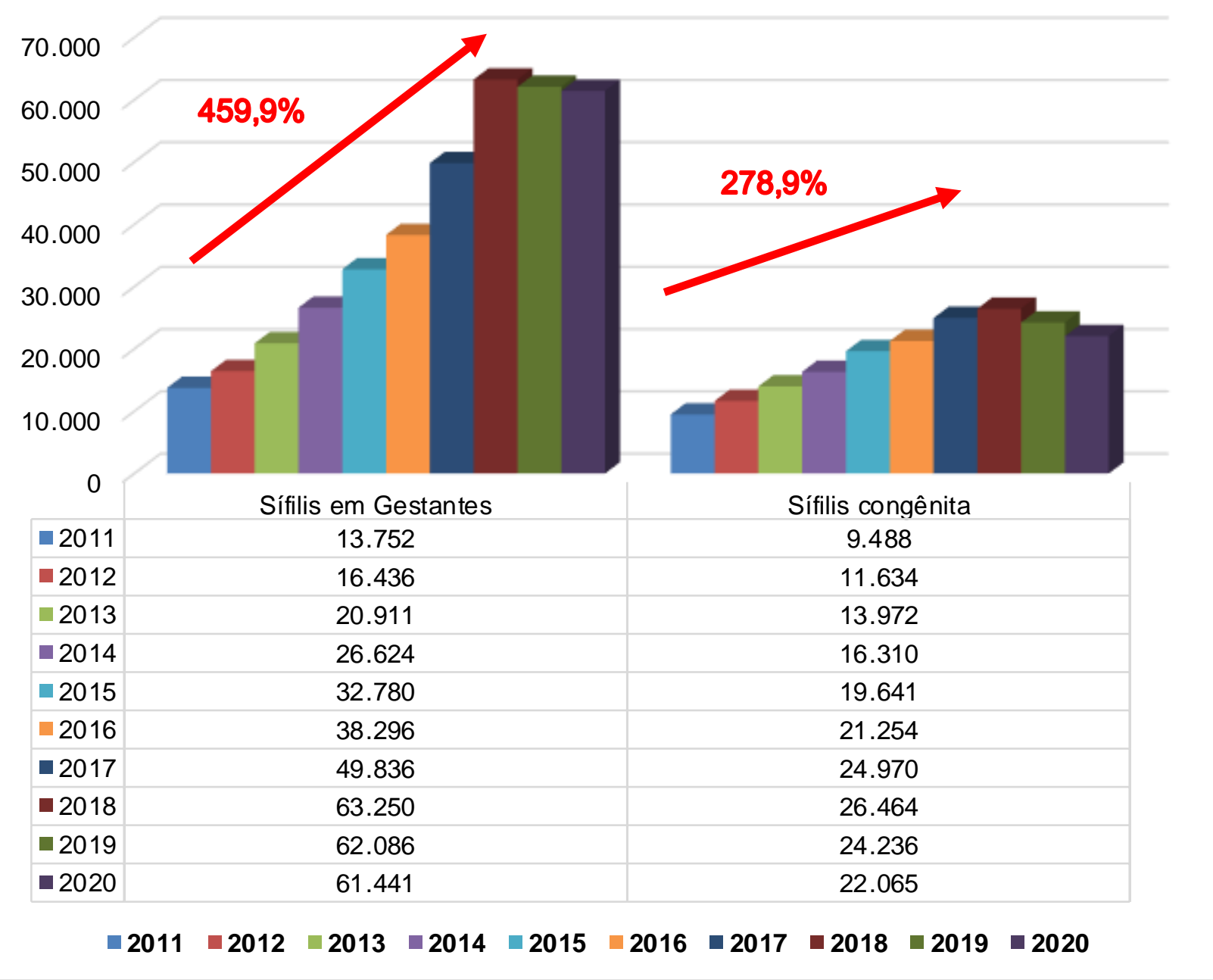

Fonte: RAMOS AM, et al., 2021; dados extraídos do Indicadores Sífilis / DCCI, 2021.

A taxa de detecção de gestantes com sífilis para cada 1.000 nascidos vivos foi de 4,7 em 2011, alcançando 21,5 em 2018, 21,8 em 2019 e 21,6 em 2020. Da mesma forma, a sífilis congênita apresentou aumento da taxa de detecção com valores de 3,3 em 2011, 8,5 em 2019 e 7,7 em 2020 (Gráfico 2). 
Gráfico 2 - Taxa de detecção (por 1.000 nascidos vivos) de sífilis em gestantes e de sífilis congênita por ano. Brasil, 2011-2020.

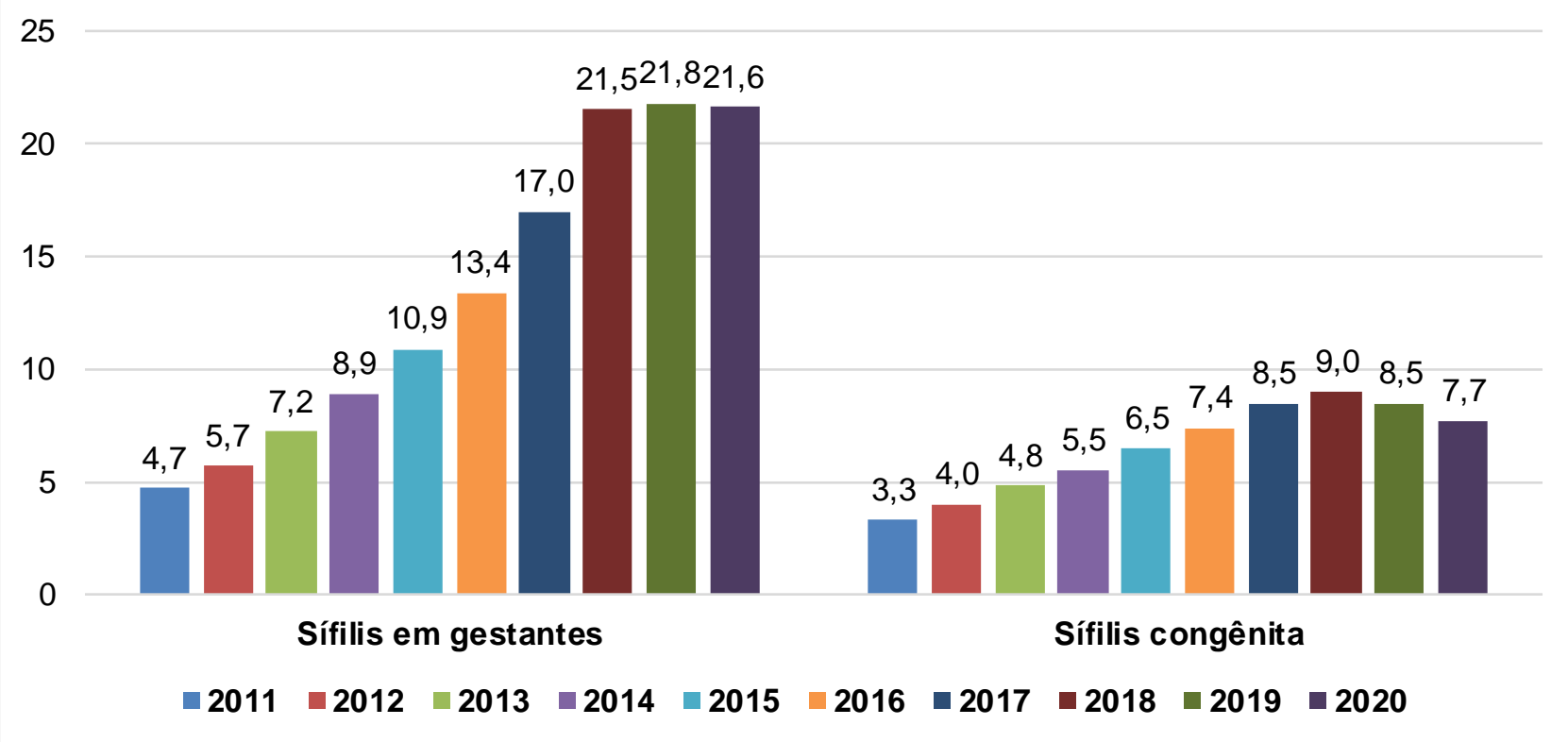

Fonte: RAMOS AM, et al., 2021; dados extraídos do Indicadores Sífilis / DCCI, 2021.

Em 2020, os estados do Acre $(30,4)$, Amazonas $(22,2)$, Mato Grosso do Sul $(30,8)$, Pernambuco $(23,2)$, Rio de Janeiro $(55,1)$, Rio Grande do Sul $(31,7)$, Roraima $(23,7)$, Sergipe $(26,2)$ e Tocantins $(24,6)$ apresentaram a taxa de detecção para sífilis em gestantes acima da taxa nacional $(21,6)$. Enquanto que outros estados apresentaram taxas bem abaixo, como Bahia $(11,4)$, Maranhão $(11,2)$, Paraíba $(12,1)$ e Piauí $(12,4)$ (Gráfico 3).

Ademais, os estados Amapá $(9,1)$, Ceará $(8,3)$, Pernambuco $(13,1)$, Rio de Janeiro $(21,5)$, Rio Grande do Norte $(12,0)$, Rio Grande do Sul $(12,9)$, Sergipe $(16,6)$ e Tocantins $(9,3)$ apresentaram taxa de detecção de sífilis congênita superiores à taxa nacional. Ao mesmo tempo em que Mato Grosso $(2,7)$, e Rondônia $(2,6)$ apresentaram as menores taxas (Gráfico 3).

Gráfico 3 - Taxa de detecção (por 1.000 nascidos vivos) de sífilis em gestantes por estados. Brasil, 2020.

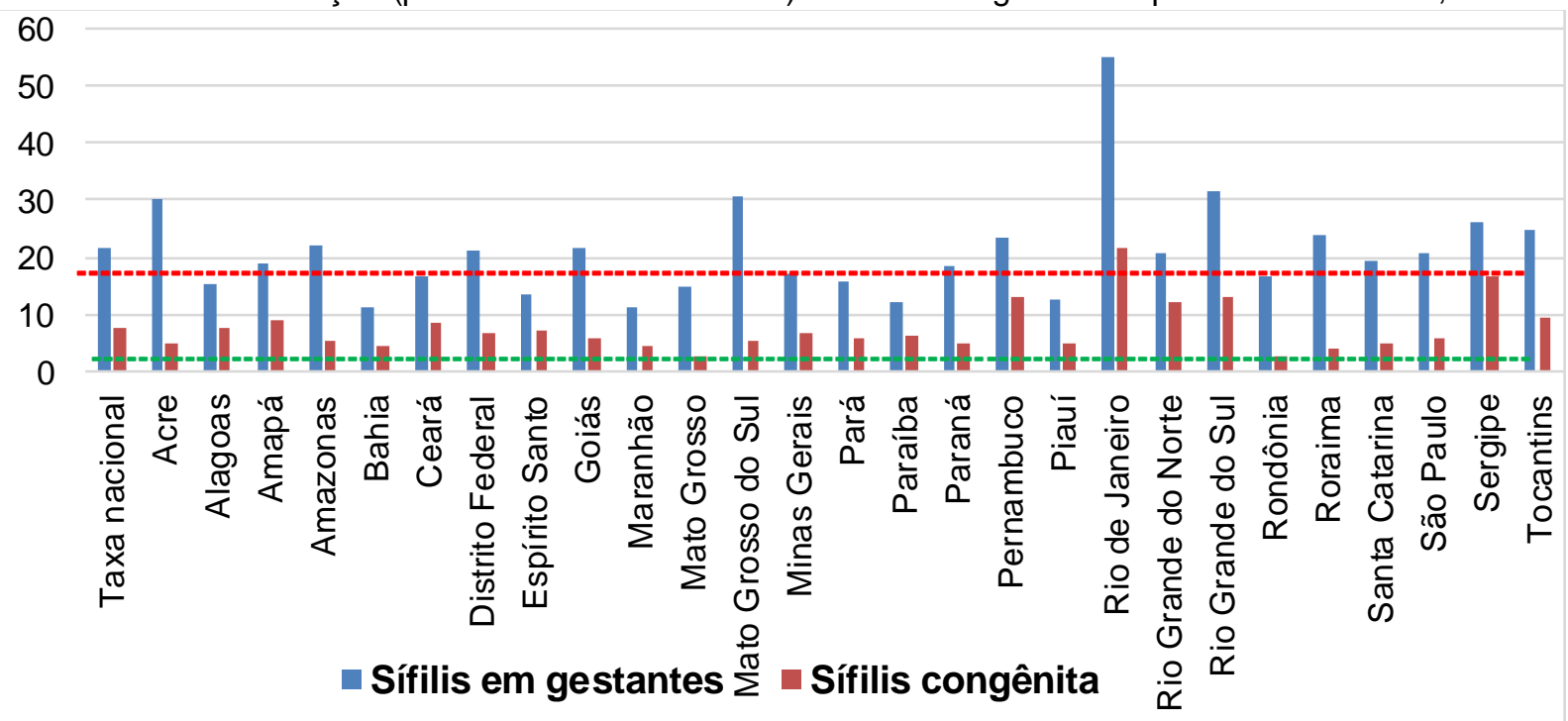

Fonte: RAMOS AM, et al., 2021; dados extraídos do Indicadores Sífilis / DCCI, 2021. 
Em relação à idade materna, foi observado um aumento do número de casos em todas as faixas etárias, em especial entre 20 e 29 anos (495,5\%), além do mais, esse grupo etário foi o mais prevalente em todos os anos em estudo, totalizando 53,4\% ( $n=205.697)$, seguido de até 19 anos com $26,1 \% \quad(n=100.543)$ e de 30 a 39 anos com $18,5 \%$ ( $n=71.309)$ (Gráfico 4$)$.

Gráfico 4 - Casos de gestantes com sífilis segundo faixa etária por ano. Brasil, 2011-2020.

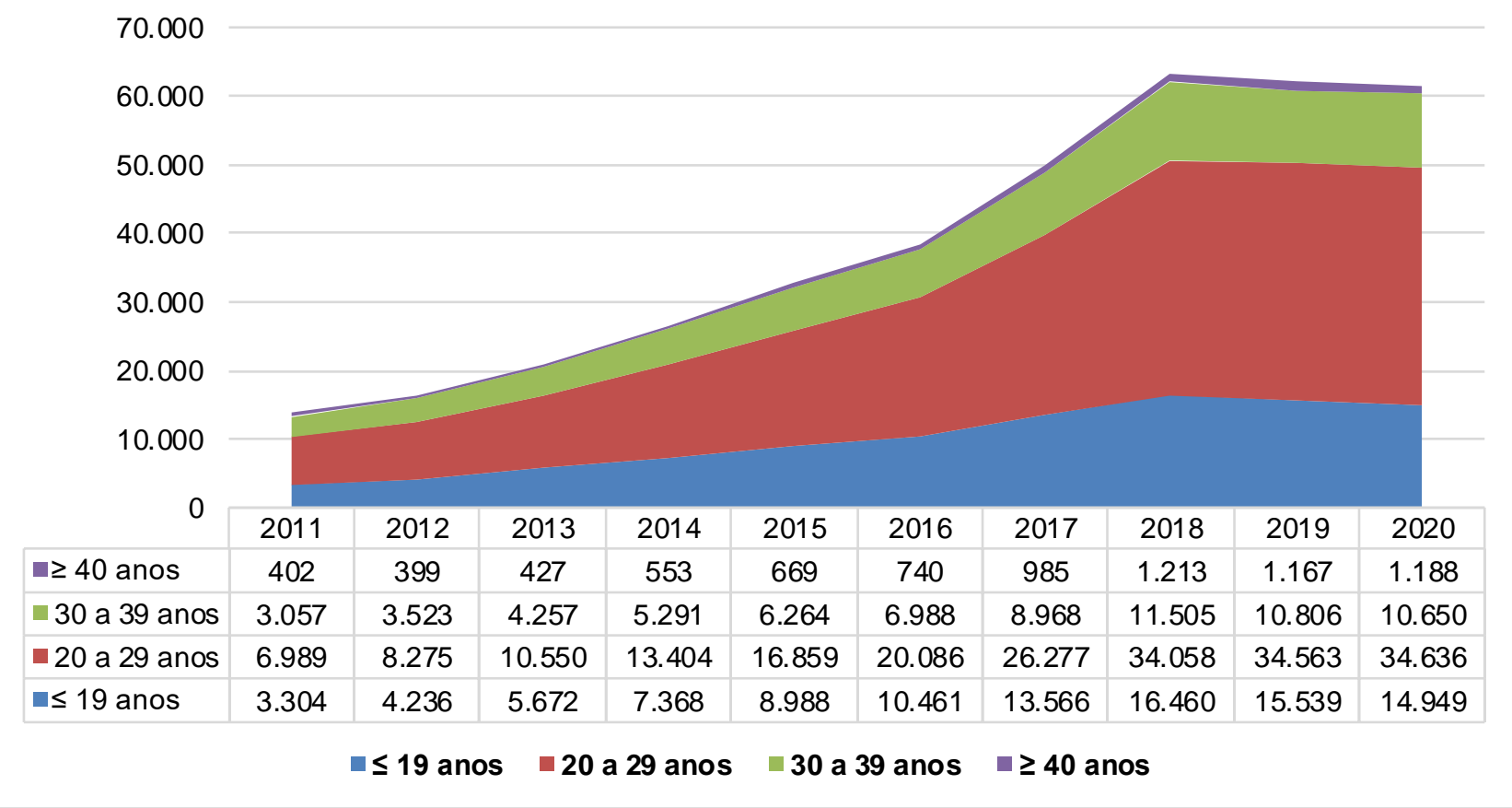

Fonte: RAMOS AM, et al., 2021; dados extraídos do Indicadores Sífilis / DCCI, 2021.

No que se refere à escolaridade, foi observado que a maior parte das gestantes com sífilis apresentou ensino fundamental incompleto $(n=94.719 ; 25,4 \%)$. Além disso, o número de casos no período estudado é menor entre as gestantes que possuem ensino superior incompleto $(n=5.102 ; 1,4 \%)$, superior completo $(n=$ 3.982; $1,1 \%$ ) e analfabetas ( $n=2.603 ; 0,7 \%$ ). Vale ressaltar que 106.507 dos casos foram classificados como ignorados, representando $28,6 \%$ da população estudada (Gráfico 5 ).

Em relação à cor/raça, a sífilis é prevalente entre pardas $(n=189.976 ; 49,3 \%)$ e brancas $(n=112.857$; $29,3 \%$ ), mantendo o mesmo padrão ao longo dos anos. Pretas, amarelas e indígenas representam juntas apenas 53.000 casos (13,7\%) (Gráfico 5 ).

Gráfico 5 - Sífilis em gestantes segundo escolaridade e raça ou cor. Brasil, 2011-2020.

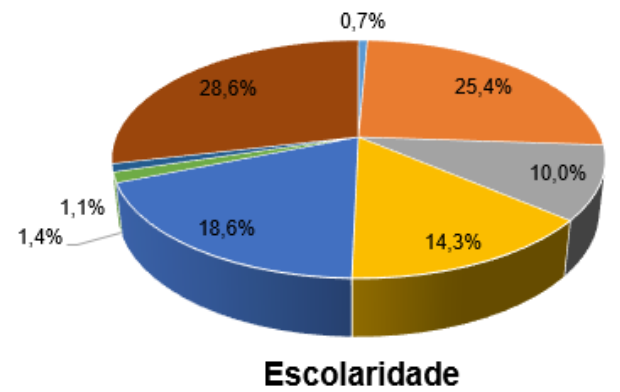

- Analfabeto

- Fundamental Completo

- Médio completo

- Superior Completo
- Fundamental incompleto

- Médio incompleto

- Superior incompleto

- Ignorado

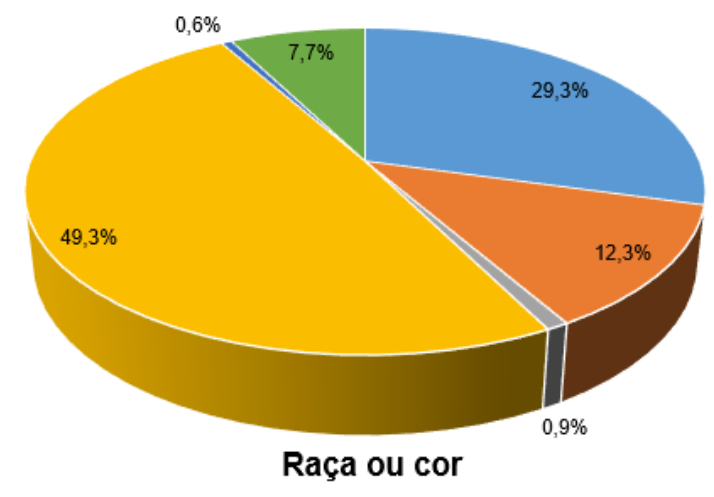

- Branca " Preta - Amarela " Parda - Indígena " Ignorada

Fonte: Ramos AM, et al., 2021; dados extraídos do Indicadores Sífilis / DCCl, 2021. 
O maior número de casos diagnosticados de sífilis em gestantes foi no primeiro trimestre $(n=138.938$; $36,1 \%$ ). No entanto, foi identificada uma inversão do padrão de detecção dos casos, uma vez que de 2011 a 2014 a maioria dos diagnósticos foram realizados no terceiro trimestre. A partir de então, houve um aumento considerável de diagnóstico no primeiro trimestre, sendo responsável por 23,1\% em 2011 e 41,8\% em 2020. Assim como, houve uma diminuição dos casos diagnosticados no $2^{\mathrm{a}}$ e $3^{\circ}$ trimestre e com idade gestacional ignorada (Gráfico 6).

Da mesma forma, a classificação da sífilis em gestantes analisada durante os 10 anos em estudo, identificou que a fase primária foi mais frequente $(n=108.832 ; 31,1 \%)$. A fase clínica da sífilis foi considerada ignorada em $26,9 \%$ ( $n=94.113)$, seguida pela fase latente $(n=91.704 ; 26,7 \%)$. Porém, foi observado que de 2011 a 2016 a maioria de casos eram de sífilis primária, ocorrendo a inversão a partir de 2017, quando a sífilis latente passou a ser mais prevalente, representando $39,8 \%(n=24.440)$ dos 61.441 casos diagnosticados em 2020. Ademais foi identificada uma diminuição das notificações da fase clínica considerada ignorada no decorrer do período em estudo (Gráfico 6).

Gráfico 6 - Distribuição percentual de casos de sífilis em gestantes segundo idade gestacional e classificação clínica por ano. Brasil, 2011-2020.
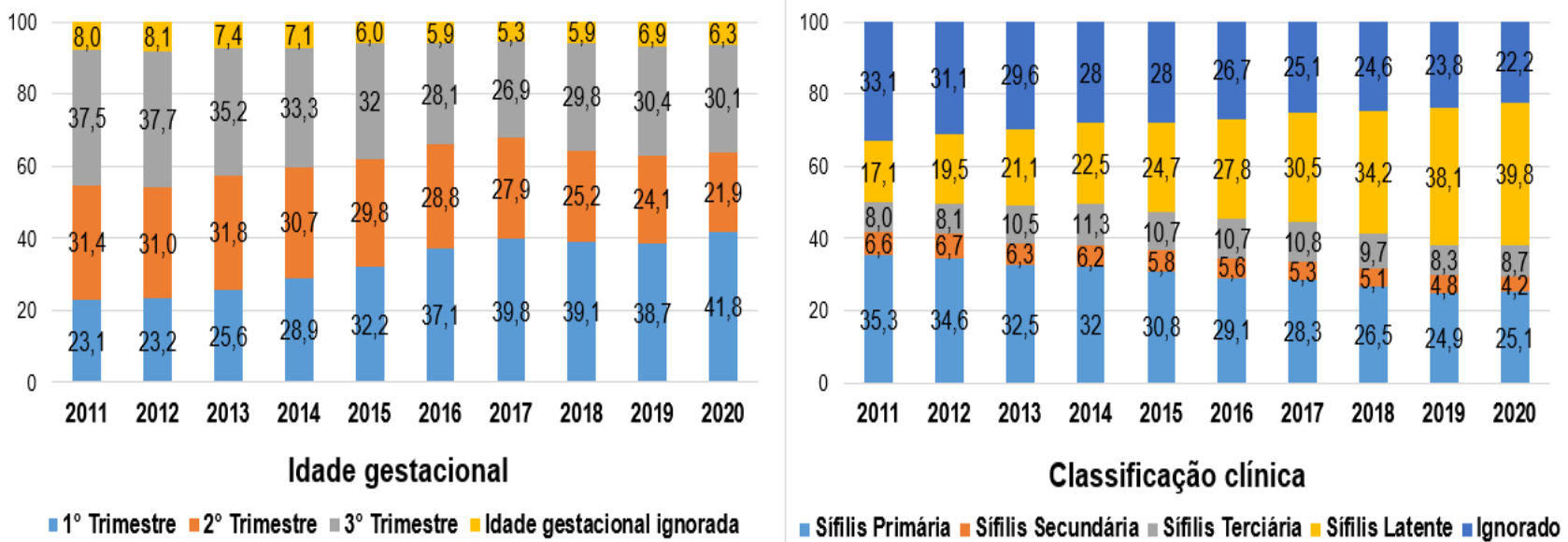

$\backsim 1^{\circ}$ Trimestre $\backsim 2^{\circ}$ Trimestre $\llbracket 3^{\circ}$ Trimestre $\llbracket$ Idade gestacional ignorada

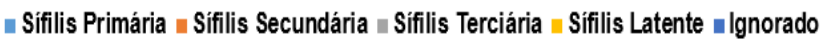

Fonte: RAMOS AM, et al., 2021; dados extraídos do Indicadores Sífilis / DCCl, 2021.

Quanto ao tratamento, estão disponíveis para análise somente dados referentes ao período de 2017 a 2020 , e os mesmos demonstram que a maioria das gestantes tratadas fizeram uso da penicilina $(n=212.270$; $89,8 \%$ ). Outros esquemas foram usados em 3.683 casos (1,5\%). Ademais, nos chama atenção o fato de que, no período estudado, 12.237 gestantes não receberam tratamento, o equivalente a $5,2 \%$ dos casos.

\section{DISCUSSÃO}

Apesar de que no período de 2012 a 2016 houve uma redução de sífilis congênita no mundo e uma estabilidade do número de casos de sífilis em gestantes (KORENROMP EL, et al., 2019). Neste estudo, foi observado um aumento da prevalência de sífilis em gestantes nos últimos anos no Brasil, o que aponta para uma situação alarmante de saúde pública. Além disso, Miraglia E, et al. (2020) também refere um aumento de casos em toda América Latina, devido ao fato desses países comungarem das mesmas características sociais. Tais locais of erecem infraestrutura e serviços públicos limitados, sendo esse cenário favorável a situações de risco, o que gera um impacto negativo sobre os indicadores de saúde (SOUZA RL, et al., 2020).

No Brasil, esse aumento de casos tem sido demonstrado em estudos de vários locais no país, como: Tocantins, São Paulo, Goiás, Pará e Fortaleza, que apontaram uma prevalência da sífilis em gestantes adultas jovens, pardas, com baixo-médio índice de escolaridade, que realizaram pré-natal de forma precária e com tratamento inadequado, concordando com os resultados do presente estudo (CAVALCANTE PAM, et al., 2017; CARDOSO ARP, et al., 2018; NUNES OS, et al., 2018; CESAR JÁ, et al., 2020; LUPPI CG, et al., 2020; SOUZA RL, et al., 2020). 
Entretanto, apesar desse aumento demonstrado, o número de notificações pode ainda ser subestimado, visto as falhas de notificação compulsória, o baixo nível de esclarecimento sobre a necessidade do pré-natal e dos cuidados durante a gestação, além de baixo acesso ao pré-natal e aos testes de rastreio (CESAR JA, et al., 2020; JOSEPH DAVEY DL, et al., 2020; BARBOSA MDS, et al., 2021).

Foi possível identificar um aumento gradativo das notificações de sífilis em gestantes e de sífilis congênita no Brasil, com uma discrepância dessas taxas de acordo com os estados brasileiros, com destaque para o Rio de Janeiro com a maior taxa em gestantes e também de sífilis congênita.

Segundo Câmara LS, et al. (2020), o aumento de casos de sífilis em gestantes no Rio de Janeiro não é somente resultante do aumento de casos, mas também da ação da vigilância epidemiológica do município, com diminuição da subnotificação e ampliação da cobertura de Estratégias de Saúde da Família, com aumento da disponibilidade dos testes rápidos e do tratamento na atenção básica.

De acordo com Figueiredo DCMM, et al. (2020), o aumento da taxa de diagnóstico de sífilis em gestantes nem sempre é acompanhada pelo tratamento oportuno e redução de sífilis congênita, situação observada na maioria dos estados brasileiros no presente estudo. Além do mais, relatam variação regional do acesso ao tratamento da sífilis na atenção básica, com menor disponibilidade de Penicilina nas regiões Nordeste e Sudeste, com provável consequência na incidência de sífilis congênita.

Foi observado que o estado do Mato Grosso do Sul apresentou uma elevada taxa de sífilis em gestantes, entretanto a de sífilis congênita menor do que a nacional, o que pode ser resultante de maior acesso aos testes sorológicos de diagnóstico e ao tratamento ef etivo das gestantes e, por conseguinte, a prevenção da transmissão vertical de sífilis.

Em suma, muitas variáveis podem interferir sobre essas notificações além da incidência da própria doença na população, como a subnotificação ou a diminuição da mesma consequente a melhorias de estratégias em saúde e a acessibilidade aos serviços em saúde como pré-natal, testes sorológicos para o diagnóstico e o tratamento eficaz.

Segundo o Boletim Epidemiológico de Sífilis do Ministério da Saúde (2020), a segunda faixa etária mais prevalente desde 2005 era de 30 a 39 anos, seguida por 15 a 19 anos, porém houve uma inversão desde 2011, ano inicial do presente estudo. Ressaltando o predomínio de gestantes jovens com sífilis nos tempos atuais. Assim como, em 2019 , foi mais frequente em gestante pardas $(51,2 \%)$ e brancas $(28,6 \%)$, em concordância com os nossos dados.

Há uma associação entre as altas taxas de prevalência de sífilis na gestação e os serviços de pré-natal inadequados (BENEDETTI KCSV, et al, 2019; BENZAKEN AS, et al, 2020). Da mesma forma Bezerra MLMB, et al. (2019) relatam o aumento de casos de sífilis congênita e aumento da mortalidade perinatal e infantil associada à sífilis em todas as regiões do Brasil. Assim como a relação da natimortalidade $c$ ausada pela sífilis e a assistência pré-natal inadequada.

É de extrema importância para a redução da sífilis congênita o rastreamento e o tratamento precoces da sífilis na assistência pré-natal, além do monitoramento das gestantes diagnosticadas e de seus recémnascidos (KORENROMP EL, et al., 2019). Segundo Torres RG, et al. (2019) a qualidade do pré-natal tem papel decisivo na prevenção vertical da sífilis.

Foi observado maior número de casos notificados no primeiro trimestre da gestação nos últimos anos, o que pode significar que uma parcela das gestantes está iniciando mais precocemente o pré-natal e, assim, fazendo o rastreio precoce da sífilis, apesar do baixo seguimento (BARBOSA MDS, et al., 2021). Quanto à classificação da sífilis, atualmente, a maior prevalência no diagnóstico é de sífilis latente, ratificando a importância dos testes de rastreio. Além do mais, dificilmente o diagnóstico de sífilis é realizado na fase primária devido à dificuldade da identificação da lesão primária na mulher (CARDOSO ARP, et al., 2018; LAFETÁ KRG, et al., 2016; MARQUES JVS, et al., 2018).

Nesta análise, a maior frequência do diagnóstico de sífilis no $1^{0}$ trimestre gestacional e nas fases latentes pode ser resultante de melhorias do pré-natal, ratificando a importância do mesmo, porém ainda há 
fragilidades nessa assistência identificadas pelo número crescente de casos simultâneos de sífilis congênita. Além disso, A diminuição das notificações com idade gestacional e fase clínica da sífilis como ignoradas pode ser resultante de melhorias no preenchimento das fichas de notificação compulsória (MS, 2020b).

Figueiredo DCMM, et al. (2020) demostraram à intima relação entre a oferta de teste rápido para sífilis na atenção básica e o aumento de diagnóstico em gestantes, assim como a disponibilidade de Penicilina para o tratamento e a redução da incidência de sífilis congênita.

O tratamento da sífilis em gestantes recomendado pela Organização Mundial de Saúde é feito com Penicilina Benzatina pela via intramuscular (WORLD HEALTH ORGANIZATION, 2017). Tal medicação é a única ef etiva no tratamento materno e simultaneamente na prevenção da transmissão vertical. Gestantes alérgicas devem ser dessensibilizadas para uso da penicilina, tal a importância do uso desta medicação. Consequentemente, as gestantes que utilizarem outras alternativas terapêuticas serão consideradas como inadequadamente tratadas. Muitas gestantes apesar de utilizarem a medicação, fazem uso de forma incorreta, abandonam o tratamento, não fazem o controle de cura recomendado e/ou não fazem o tratamento do parceiro concomitante, com consequente reinfecção (NONATO SM, et al., 2015).

Para ser considerado adequado o tratamento de sífilis em gestantes deve ser realizado esquema completo da penicilina Benzatina, de acordo com o estágio clínico, iniciada pelo menos 30 dias antes do parto. É de grande importância a verificação do intervalo entre as doses e o risco de reinfecção, além do controle de cura com a queda da titulação do teste não treponêmico em pelo menos duas diluições em três meses ou de quatro diluições em seis meses após o término do tratamento. Geralmente o diagnóstico de sífilis em gestantes ocorre na fase latente e como não se pode definir a cronologia da inf ecção deve ser trat ada como sífilis latente tardia (MS, 2018).

Apesar do uso da medicação Penicilina Benzatina na maioria das gestantes com sífilis foi observado um crescimento do número de casos de sífilis congênita, pode ser reflexo de um pré-natal deficiente, com baixo seguimento e adesão pelas gestantes, sem acompanhamento pelo profissional médico. Tal fato explica os altos índices de sífilis congênita e outros desfechos pós-natais mesmo entre as gestantes que realizaram prénatal e que referem tratamento com Penicilina Benzatina (PADOVANI C, et al., 2018; COOPER JM e SÁNCHEZ PJ, 2018).

\section{CONCLUSÃO}

A sífilis em gestantes apresentou um aumento de casos no decorrer do período em estudo, com prevalência em mulheres jovens, pardas e de baixa escolaridade. A maior frequência do diagnóstico de sífilis no $1^{\circ}$ trimestre gestacional e nas fases latentes pode ser resultante de melhorias do pré-natal, porém o crescimento simultâneo de casos de sífilis congênita ratifica fragilidades dessa assistência materna. Este estudo é uma ferramenta para mudar o cenário atual, uma vez que é possível identificar a necessidade de investimentos em saúde pública na área, favorecendo o diagnóstico precoce e o tratamento adequado da gestante e do parceiro para reduzir as complicações da sífilis no binômio materno-fetal.

\section{REFERÊNCIAS}

1. BARBOSA MDS, et al. Epidemiological study in Brazilian women highlights that syphilis remains a public health problem. Revista do Instituto de Medicina Tropical de São Paulo, 2021; 63: e4.

2. BARBOSA DRM, et al. Perfil Epidemiológico dos Casos de Sífilis em Gestantes Brasileiras entre 2016 e 2018. Revista Interdisciplinar em Saúde, 2018;5(6): 1652-1668.

3. BENEDETTI KCSV, et al. High Prevalence of Syphilis and Inadequate Prenatal Care in Brazilian Pregnant Women: A Cross-Sectional Study. American Journal of Tropical Medicine and Hygiene, 2019;101(4): 761-766.

4. BENZAKEN AS, etal. Adequacy of prenatal care, diagnosis and treatment of syphilis in pregnancy: a study with open data from Brazilian state capitals. Caderno de Saúde Pública, 2020;36(1): e00057219.

5. BEZERRA MLMB, et al. Congenital Syphilis as a Measure of Maternal and Child Healthcare, Brazil. Emerging Infectious Diseases, 2019;25(8): 1469-1476.

6. CÂMARA LS, et al. Perfil epidemiológico das gestantes com sífilis no Rio de Janeiro. Research, Society and Development, 2020;9(10):e4999108712. 
7. CARDOSO ARP, et al. Análise dos casos de sífilis gestacional e congênita nos anos de 2008 a 2010 em Fortaleza, Ceará, Brasil. Ciências e Saúde Coletiva, 2018;23(2):563-574.

8. CAVALCANTE PAM, et al. Sífilis gestacional e congênita em Palmas, Tocantins, 2007-2014. Epidemiologia e Serviço de Saúde, 2017;26(2): 255-264.

9. CESAR JA, et al. Não realização de teste sorológico para sífilis durante o pré-natal: prevalência e fatores associados. Revista Brasileira de Epidemiologia, 2020;23: e200012.

10. CONCEIÇÃO HN, et al. Análise epidemiológica e espacial dos casos de sífilis gestacional e congênita. Saúde debate, 2019;43(123);1145-1158.

11. COOPER JM, SÁNCHEZ PJ. Congenital syphilis. Seminars in Perinatology, 2018;42(3):176-184.

12. DANTAS LA, et al. Perfil epidemiológico de sífilis adquirida diagnosticaday notificada en hospital universitario $m$ atemo infantil. Enferm. glob., 2017;16(46):217-245.

13. FIGUEIREDO DCMM, et al. Relação entre oferta de diagnóstico e tratamento da sífilis na atenção básica sobre a incidência de sífilis gestacional e congênita. Caderno de Saúde Pública, 2020;36(3): e00074519.

14. JOSEPH DAVEY DL, et al. Prevalence of Curable Sexually Transmitted Infections in Pregnant Women in Low - and Middle-Income Countries From 2010 to 2015: A Systematic Review. Sexually transmitted diseases, 2016;43(7): 4508.

15. KORENROMP EL, et al. Global burden of maternal and congenital syphilis and associated adverse birth outcomesEstimates for 2016 and progress since 2012. PLoS One, 2019;14(2): e0211720.

16. LAFETÁ KRG, et al. Sífilis materna e congênita, subnotificação e difícil controle. Revista Brasileira de Epidemiologia, $2016 ; 19(1): 63-74$.

17. LUPPI CG, et al. Syphilis in the state of São Paulo, Brazil, 2011-2017. Revista Brasileira de Epidemiologia, 2020;23: e200103.

18. MAGALHÃES DMS, et al. A sífilis na gestação e sua influência na morbimortalidade materno-infantil. Comunicação em Ciências Saúde, 2011;22 Sup 1: S43-S54.

19. MARQUES JVS, et al. Perfil epidemiológico da Sífilis Gestacional: Clínica e Evolução de 2012 a 2017. SANARE, Sobral, 2018; 17(2): 13-20.

20. MASCHIO-LIMA T, et al. Perfil epidemiológico de pacientes com sífilis congênita e gestacional em um município do Estado de São Paulo, Brasil. Revista Brasileira de Saúde Materno Infantil, 2019;19(4): 865-872.

21. MENEZES ML, PASSOS MR. Sífilis e gravidez. Federação Brasileira das Associações de Ginecologia e Obstetrícia (FEBRASGO), 2018.

22. MINISTÉRIO DA SAÚDE (MS). Boletim epidemiológico Sífilis. 2020a. Disponível em: https://www.gov.br/saude/pt$\mathrm{br} / \mathrm{media} / \mathrm{pdf} / 2020 /$ outubro/29/BoletimSfilis2020especial.pdf. Acessado em :20 de novembro de 2021.

23. MINISTÉRIO DA SAÚDE (MS). Manual técnico para o diagnóstico da sífilis. 2021. Disponível em: file://C:/Users/Admin/Downloads/manual_sifilis_final_10.11.21.pdf. Acessado:em 20 de novembro de 2021 .

24. MINISTÉRIO DA SAÚDE (MS). Protocolo Clínico e Diretrizes Terapêuticas para Atenção Integral às Pessoas com Infecções Sexualmente Transmissíveis (IST). 2020b. Disponível em http://www.aids.gov.br/pt-br/pub/2015/protocoloclinico-e-diretrizes-terapeuticas-para-atencao-integral-pessoas-com-infeccoes. Acessado em: 1 de dezembro de 2021.

25. MINISTÉRIO DA SAÚDE (MS). Protocolo Clínico e Diretrizes Terapêuticas para Prevenção da Transmissão Vertical de HIV, Sífilis e Hepatites Virais. 2018. Disponível em: http://www.aids.gov.br/pt-br/pub/2015/protocolo-clinico-ediretrizes-terapeuticas-para-prevencao-da-transmissao-vertical-de-hiv. Acessado em:20 de Agosto de 2021.

26. MIRAGLIA E, et al. Prevalencia de sífilis en un hospital de la provincia de Buenos Aires en 8 años Revista de la Facultad de Ciencias Médicas de Córdoba,2020;77 (3): 136-142.

27. NONATO SM, et al. Sífilis na gestação e fatores associados à sífilis congênita em Belo Horizonte-MG, 2010-2013. Epidemiologia e Serviços de Saúde, 2015; 24 (4): 681-694.

28. NUNES PS, et al. Sífilis gestacional e congênita e sua relação com a cobertura da Estratégia Saúde da Família, Goiás, 2007-2014:um estudo ecológico. Epidemiologia e Serviços de Saúde, 2018;27 (4): e2018127.

29. PADOVANI C, et al. Syphilis in during pregnancy: association of maternal and perinatal characteristics in a region of southern Brazil. Revista Latino-Amercano de Enfermagem, 2018;26: e3019.

30. SARACENI V, et al. Vigilância epidemiológica da transmissão vertical da sífilis: dados de seis unidades federativas no Brasil. Rev Panam Salud Publica, 2017;41: e44.

31. SILVA GM, et al. Sífilis na gestante e congênita: perfil epidemiológico e prevalência. Enfermería Global, $2020 ; 19$ (57): 107-150.

32. SOUZA RL, et al. Prevalence of syphilis in female sex workers in three countryside cities of the state of Pará, Brazilian Amazon. BMC Infectious Diseases, 2020;20 (1): 129.

33. TORRES RG, et al. Syphilis in Pregnancy: The Reality in a Public Hospital. Revista Brasileira de Ginecologia e Obstetrícia., 2019;41 (2): 90-96.

34. WORLD HEALTH ORGANIZATION (WHO). Guideline on syphilis screening and treatment for pregnant women.2017. Disponível em: https:/www.guidelinecentral.com/summaries/who-guideline-on-syphilis-screening-and-treatment-forpregnant-women/\#section-society. Acessado em :20 de outubro de 2021. 